\title{
IMECE2002-DSC-33680
}

\section{Toward improved CVTs: theoretical and experimental results}

\author{
M. E. Brokowski \\ Northwestern University
}

\author{
R. Brent Gillespie \\ University of Michigan
}

\author{
Songho Kim \\ Northwestern University
}

J. E. Colgate

Northwestern University

\author{
Michael Peshkin \\ Northwestern University
}

\begin{abstract}
Revolute-jointed cobots depend on continuously variable transmissions (CVTs). CVTs constrain the speed ratio between two shafts to a particular value within a continuous range. The spherical CVT is well suited to cobots, but like other point contact transmissions, achieving good performance is challenging.

We have studied the contact mechanics of the spherical CVT under certain simplifying assumptions. Results of the calculations are compared to experimental measurements of an instrumented CVT. Improved CVT performance may require better control of compliance and contact forces in the CVT. Results for the contact forces under load conditions are shown. We conclude with a discussion of design implications for improved CVTs.
\end{abstract}

\section{INTRODUCTION}

\subsection{Continuously Variable Transmissions (CVTs)}

A continuously variable transmission $(C V T)$ is a mechanical transformer whose modulus can be programmed to any value on a continuous range. The modulus is the speed ratio between two velocities. These might be the rotational velocities $\omega_{1}$ and $\omega_{2}$ of two drive shafts in a rotational CVT or two linear velocities $v_{\mathrm{x}}$ and $v_{\mathrm{y}}$ in a translational CVT.

A spherical CVT design exists [8] utilizing rolling contacts between a sphere, a set of drive rollers, and a set of steering rollers. The steering rollers control the speed ratio between the drive rollers by varying a transmission angle, ideally allowing the spherical CVT a speed ratio on the range $(+\infty,-\infty)$. This spherical CVT is a core element of a novel class of collaborative robotic devices called cobots whose end effector paths are constrained by CVTs at their joints to travel only along trajectories programmed by tuning the speed ratios of the CVTs [1][2].

\subsection{Other Work}

Goi, Kawakami, Yamakawa, and Tanaka[3] discuss the design and testing of a helicopter traction-drive CVT in high viscous traction fluid. The CVT they design allows for output speeds between $85 \%$ and $100 \%$ of the input speed (roughly 23,000 RPM) and tested over input torques up to $40 \mathrm{Nm}$.

Kluger and Fussner [4] review several CVT mechanisms and efficiencies, including belt type, hydrodynamic traction type, and epicycle type mechanisms. They typically find efficiencies greater than $85 \%$ for each design they examine.

Singh and Nair [5] also review a variety of CVT designs and compare their relative efficiencies after "normalizing" the operating ranges of the different CVT types. They include in their survey belt type, and hydrodynamic traction type CVTs along with hydrodynamic torque converters.

(Neither 4] nor [5] examine a CVT design based on dry rolling contact.)

The device considered in this work is the spherical CVT depicted conceptually in Figure 2. Johnson [6] reviews rolling contact models, which underlie the mechanics of the rolling contact patch between both the drive and steering rollers and the sphere in a spherical CVT.

Moore [7] and Moore, Peshkin, and Colgate B] have described the spherical CVT in the context of its use as a nonholonomic element in serial link cobots. Moore discusses (among other things) CVT design goals for use in cobots and performs experiments on a particular CVT to determine maximum supportable torques for that CVT.

Gillespie, Peshkin, Colgate, and Moore 9] develop an analytical model to examine the effects of longitudal kinematic creep and spin in the contact patches of the drive rollers of a spherical CVT. They determine an expression that predicts the speed ratio of a spherical CVT at a particular transmission angle in the face of a lateral torque. They also review a simple translational CVT (an elastic wheel rolling on a plane) and discuss the notion of a sideslip angle when that 
CVT is subject to lateral forces. They develop an expression for an analogous sideslip angle in the spherical CVT. It is the analytical creep and spin model developed in 9] which the present work uses for comparison with experimental results.

\subsection{Present Work}

As we might expect, real CVTs cannot achieve speed ratios on the range $(+\infty,-\infty)$ when transmitting loads, due to various losses in the rolling contacts. The spherical CVT has been subject to analysis by Gillespie et alia [9] which models kinematic creep and spin in the contact patches of the drive rollers in the presence of lateral loading and which predicts slip that varies with the transmission angle and the applied lateral load and results in a departure from the ideal speed ratio.

The present work subjects a physical CVT to experimental testing to determine the ability of the creep/spin model to predict the speed ratio for the transmission. We introduce a bond graph model of the spherical CVT, accounting for both the kinematic creep and spin losses analyzed in [9] and various other frictions we model for experimental computation. We determine the speed ratio of the actual CVT under various lateral loads compare that to the predictions of the creep/spin model.

In addition, we test the utility of the creep/spin model to allow corrective resteering of the CVT transmission angle to ensure that it provides the desired speed ratio under a known lateral load and we compare this to resteering attempts made by interpolating within the collected data.

We also summarize investigation of transmission losses in the spherical CVT due to creep and spin. We present a bond graph model incorporating these losses and use it to determine the CVT's power transmission efficiency, $\eta$, predicted by the model and compare it to the total efficiency as measured by experiment.

We find that the creep/spin model fits the form of the collected data quite well, though it tends to underpredict overall losses. However, by fitting a model parameter to observed data, the creep/spin model can be practically used to resteer CVTs.

\subsection{Assumptions}

This work considers only the spherical CVT in dynamic equilibrium (i.e. constant velocity of both flows coupled by the CVT). We do not consider transient effects of either acceleration of either drive roller or reorientation of the steering rollers. See Section 6 for consideration of other assumptions.

\subsection{Organization}

Section 2 reviews ideal CVT motion for translational and rotational CVTs and summarizes the results of the kinematic creep and spin model to which the experimental data will be compared. Section 3 describes the experimental hardware and software setup used in this work and introduces the bond graph model we use to model the spherical CVT. Section 4 discusses the reduced experimental data in terms of sideslip angle and speed ratio and compares it to the predictions of the creep/spin model. We also discuss the theoretical and measured power efficiencies of the CVT. Section 5 summarizes the resteering experiments used to show the utility of the creep/spin model in pre-correcting the CVT in the face of a known lateral torque. Section 6 discusses some of the outstanding issues in CVT design and possible directions for research to improve the next generation of CVTs. Section 7 summarizes our findings and concludes this work.

\section{CVT MOTION}

\subsection{Nominal CVT Motion}

Our CVTs, both translational and rotational, have an allowed direction of motion determined by contact geometry. In general, it is simpler to think in terms of the translational CVT, but the behavior of the spherical CVT is analogous.

\subsubsection{Translational CVT}

Figure 1 depicts a top-view of a simple translational CVT, an upright disk (wheel) rolling on a planar surface.

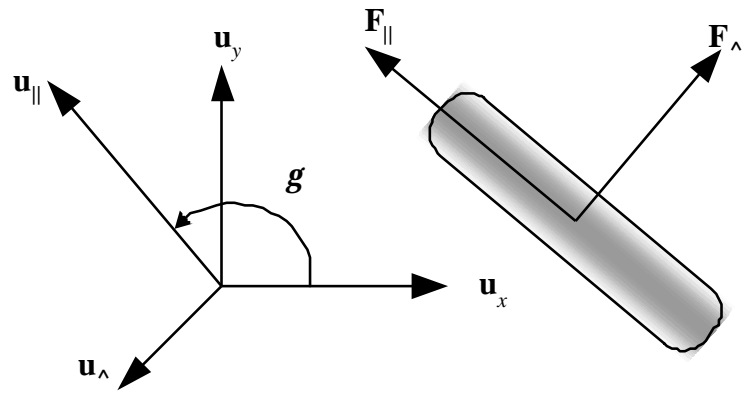

Figure 1. Top-view of a simple, translational CVT: a disk rolling in a plane

As we move the disk along the $x$-axis, its rolling constraint provides motion along $y$ as well. (The $\mathbf{F}_{\perp}$ shown here will have a negative measure number.)

Nominally, the disk is free to move along the allowed $\mathbf{u}_{\|}$ direction and cannot move along the disallowed, lateral $\mathbf{u}_{\perp}$ direction without skidding. If, as shown, the disk is oriented at a steering angle, $+\gamma$ measured from the $x$-axis of the Cartesian basis $\left(\mathbf{u}_{x}, \mathbf{u}_{y}\right)$, then we can define a coordinate basis which describes the coupling between basis $\left(\mathbf{u}_{x}, \mathbf{u}_{y}\right)$ and the allowed and disallowed directions of motion, $\left(\mathbf{u}_{\|}, \mathbf{u}_{\perp}\right)$. For this basis, the unit vectors describing the allowed and lateral directions of motion are given by

$$
\begin{aligned}
& \mathbf{u}_{\|}=u_{x} \mathbf{u}_{x}+u_{y} \mathbf{u}_{y}=(\cos \gamma) \mathbf{u}_{x}+(\sin \gamma) \mathbf{u}_{y} \\
& \mathbf{u}_{\perp}=(-\sin \gamma) \mathbf{u}_{x}+(\cos \gamma) \mathbf{u}_{y}
\end{aligned}
$$

We can determine a nominal speed ratio for this translational CVT as the ratio of $y$-axis motion to $x$-axis motion. Thus,

$$
T_{y / x}=u_{y} / u_{x}=\tan \gamma
$$

For a system in equilibrium, we know that $\mathbf{F}_{\|}=\mathbf{0}$. We can apply that condition in the $\left(\mathbf{u}_{x}, \mathbf{u}_{y}\right)$ basis and determine that 


$$
f_{y} / f_{x}=-1 / \tan \gamma=-1 / T_{y / x}
$$

\subsubsection{Rotational CVT}

A similar relation applies to the spherical CVT [7][8][9], which couples the rotational velocities of two drive shafts to that of a sphere that spins between them. This coupling as accomplished by maintaining the orientation of the axis of rotation (or transmission axis) of the sphere such that each of the drive rollers is rolling along a circle of a different radius on surface of the sphere. (We refer to these two drive shaft velocities as motor and brake velocities, $\omega_{m}$ and $\omega_{b}$.)

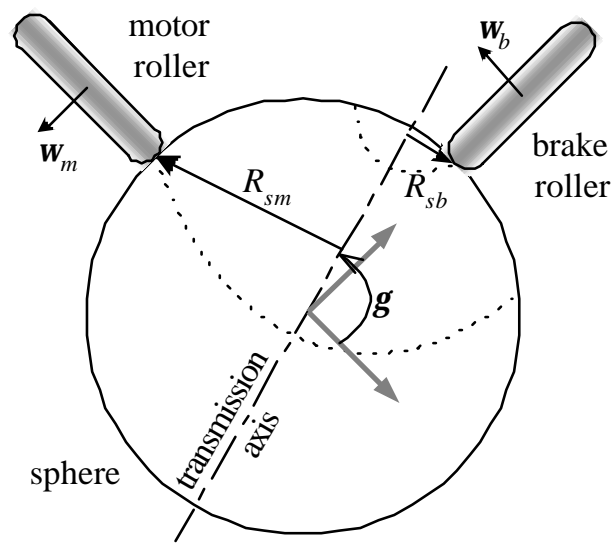

Figure 2. Simplified Spherical CVT: sphere and two drive rollers

The motor and drive rollers roll on a sphere constrained to rotate about a transmission axis. The radii of the circles on which they roll determine the drive roller speeds.

The actual implementation is somewhat involved, but Figure 2 illustrates a simplified spherical CVT, with two drive rollers in rolling contact with a sphere. The motor and brake rollers roll on circles of radius $R_{s m}$ and $R_{s b}$ respectively. We can determine the ratio of motor speed to brake speed:

$$
T_{m / b}=\omega_{m} / \omega_{b}=\left(\omega_{m} / \omega_{s}\right)\left(\omega_{s} / \omega_{b}\right)=R_{s m} / R_{s b}
$$

By changing the transmission angle $\gamma$, we can set the radii on which the drive rollers rolls anywhere on the range $[0, R]$, where $R$ is the radius of the CVT sphere. The radii of the circles on which the motor and brake rollers roll can be shown to be

$$
\begin{aligned}
& R_{s m}=R \sin \gamma \text { and } \\
& R_{s b}=R \cos \gamma
\end{aligned}
$$

And we can substitute (6) into (5) and find the speed ratio in terms of $\gamma$

$$
T_{m / b}=\omega_{m} / \omega_{b}=\tan \gamma
$$

just as it was for the translational CVT.

Equations (6) also make clear that $R_{s m}$ and $R_{s b}$ may assume zero and then negative values as $\gamma$ crosses multiples of $\pi / 2$. In this way the spherical CVT can (ideally) achieve speed ratios from $-\infty$ to $\infty$.

When examining the translational CVT, we noted that the transmission angle couples the Cartesian basis with an allowed/disallowed basis. We can define the transmission angle for the spherical CVT and develop a set of bases that describe both the motor and brake velocities and the allowed and disallowed velocities. Figure 3 shows those bases in quadrant I, where $0<\gamma<\pi / 2$.

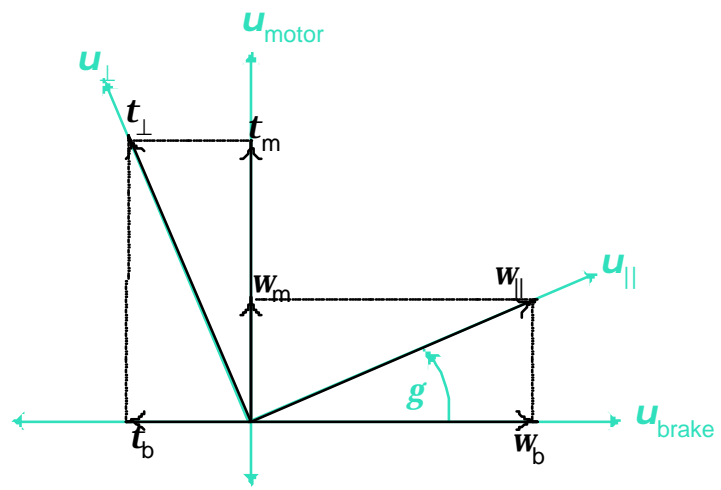

Figure 3. Bases for a Spherical CVT, quadrant I

Here we note that the speeds of the motor and brake shafts, given by $\omega_{m}$ and $\omega_{b}$, respectively, are the measure numbers for the $\left(\mathbf{u}_{\text {brake }}, \mathbf{u}_{\text {moto }}\right)$ basis and they correspond to the Cartesian $x$ and $y$ velocities in the translational CVT.

Figure 3 as sumes that we have commanded a positive velocity from the motor, placing $\omega_{\mathrm{m}}$ on the positive $\mathbf{u}_{\mathrm{motor}}$ axis. The $\left(\mathbf{u}_{\|}, \mathbf{u}_{\perp}\right)$ basis is rotated $+\gamma$ from the $\left(\mathbf{u}_{\text {brake }}, \mathbf{u}_{\text {motor }}\right)$ basis, so we can follow $\omega_{\mathrm{m}}$ over to the $\mathbf{u}_{\|}$axis (where we see $\omega_{\|}$is positive) and then down to find that $\omega_{b}$ lies on the positive half of the $\mathbf{u}_{\text {brake }}$ axis. For dissipative brakes, $\tau_{b}$ must lie on the negative side of that axis. As a check, following $\tau_{\mathrm{b}}$ up to where it intersects $\mathbf{u}_{\perp}$ (giving us a positive $\tau_{\perp}$ ) and then back to the $\mathbf{u}_{\text {motor }}$ axis shows $\tau_{\text {motor }}$ to be positive, as it must be with a positive $\omega_{\mathrm{m}}$ to contribute power to the system.

We note that, similar to the translational CVT,

$$
\omega_{\mathrm{m}} / \omega_{\mathrm{b}}=\tan \gamma
$$

And, for systems in equilibrium, $\tau_{\|}=\mathbf{0}$. Thus,

$$
\tau_{\mathrm{m}} / \tau_{\mathrm{b}}=-1 / \tan \gamma
$$

We could als o derive (9) as a consequence of conservation of energy (power) in this lossless, ideal CVT. The equilibrium condition also relates the lateral torque load $\tau_{\perp}$ to $\tau_{\mathrm{m}}$ and $\tau_{\mathrm{b}}$ so that

$$
\begin{aligned}
& \tau_{\perp}=\tau_{\mathrm{m}} / \cos \gamma \\
& \tau_{\perp}=-\tau_{\mathrm{b}} / \sin \gamma
\end{aligned}
$$

\subsection{CVT Slip}

\subsubsection{Translational CVT Slip}

For a real disk, when the applied forces contain components lateral to the nominal path, these lateral forces will cause the disk to slip somewhat and leave its nominal path, as shown in Figure 4. 


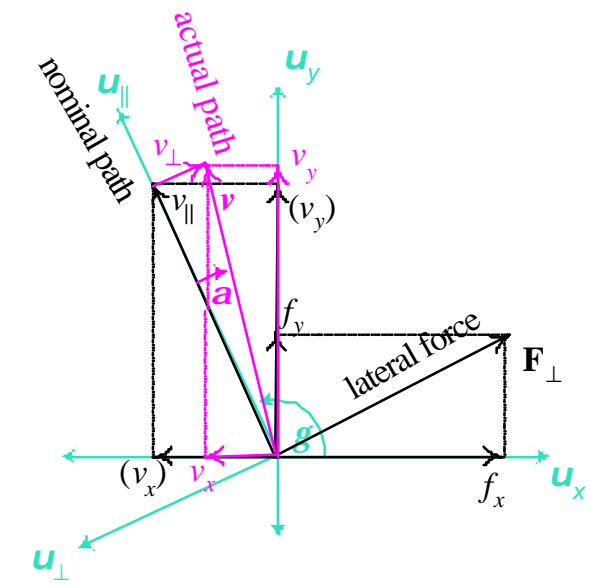

Figure 4. Actual direction of motion for rolling disk with lateral force

This new direction of motion deviates from the nominal direction of motion by a sideslip angle, $\alpha$. (Nominal quantities shown in parentheses.)

The rolling disk running as depicted in Figure 1 will tend to deviate from its nominal velocity. Figure 4 shows such a disk following a path shifted from its nominal path by a sideslip angle $\alpha$ measured clockwise from the allowed direction of motion, so $\alpha<0$ for the case shown. (The measure numbers $v_{x}$ and $v_{y}$ for the $\mathbf{u}_{x}$ and $\mathbf{u}_{y}$ velocities for the ideal case with no slip are shown in parentheses for reference.) As we would expect for the lateral force shown, the resulting $v_{x}$ and $v_{y}$ measure numbers are more positive than before.

Considering motion with the given sideslip angle, then, our speed ratio $T$ is now given by

$$
T_{y / x}=u_{\mathrm{y}} / u_{\mathrm{x}}=\tan (\gamma+\alpha)
$$

Though motion changes, the same kinetic equilibrium condition applies as in the no slip CVT,

$$
f_{y} / f_{x}=-1 / \tan \gamma
$$

\subsubsection{Rotational CVT Slip}

As with a translational CVTs, the spherical CVT will exhibit some slip when subject to lateral torques. In the conceptual spherical CVT of Figure 2, this is equivalent to the motor and brake rollers slipping slightly in their rolling contact with the sphere. While the contact mechanics details of the slip in spherical CVTs differ significantly from those of the thin wheel, the resulting slip is analogous.

In Figure 5, we can see that the spherical CVT has a $\tau_{\perp}$ in place of the translational CVT's $f_{\perp}$ and the $\left(\mathbf{u}_{\|}, \mathbf{u}_{\perp}\right)$ basis for the spherical CVT corresponds to $\left(\mathbf{u}_{\|}, \mathbf{u}_{\perp}\right)$ for the translational CVT. In the same sense for both CVTs, efforts along $\mathbf{u}_{\|}$tend to accelerate the system along its allowed direction of motion and efforts along $\mathbf{u}_{\perp}$ cause deviation from that motion.

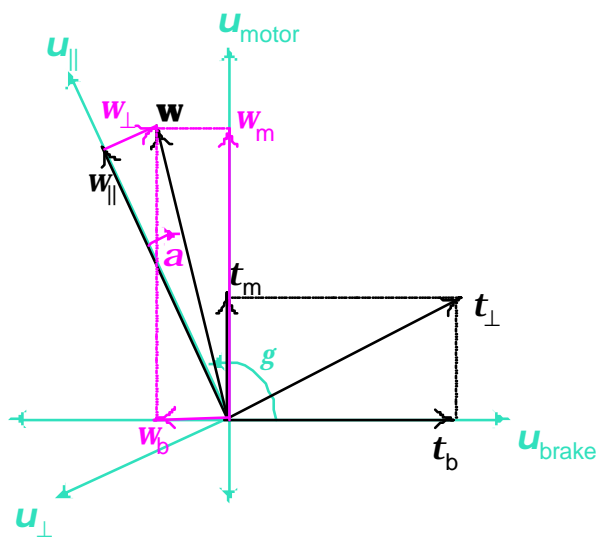

Figure 5. Motor/brake and allowed/disallowed bases for a spherical CVT

As with the translational CVT, the actual speed ratio differs from the nominal ratio. Gillespie 9] has modeled kinematic creep and spin in the drive roller contact patches of a spherical CVT and determined an expression for the actual speed ratio at a given transmission angle in the face of lateral loads. He finds the ratio of the motor-to-brake velocities to be

$$
\begin{aligned}
& T_{m / b}=\frac{\sin (\gamma)-\tau_{\perp} k \cos ^{2}(\gamma)}{\cos (\gamma)+\tau_{\perp} k \sin ^{2}(\gamma)} \quad\left(0<\gamma<\frac{\pi}{2}\right) \\
& T_{m / b}=\frac{\sin (\gamma)+\tau_{\perp} k \cos ^{2}(\gamma)}{\cos (\gamma)+\tau_{\perp} k \sin ^{2}(\gamma)} \quad\left(-\frac{\pi}{2}<\gamma<0\right)
\end{aligned}
$$

Where $\tau_{\perp}$ is the lateral torque load defined in (10) and (11) and $k$ is a constant we have amalgamated based on the specific CVT design and material parameters (described in section 3.1.1). Note that, when $\tau_{\perp}$ is zero, the creep/spin model described by (14) and (15) is the same as the ideal model given by (9). Figure 6 shows brake speed over motor speed predictions as transmission angle $\gamma$ varies for the ideal model and creep/spin model predictions for two values of $k \tau_{\perp}$.

As we might expect, the creep/spin model predicts that larger lateral torque loads cause larger deviations from ideal behavior as more slip occurs at the drive rollers.

In addition, we can define the sideslip angle in a spherical CVT in a way completely analogous to that given in (12). Thus,

$$
T_{m / b}=\frac{\omega_{m}}{\omega_{b}}=\tan (\gamma+\alpha)
$$




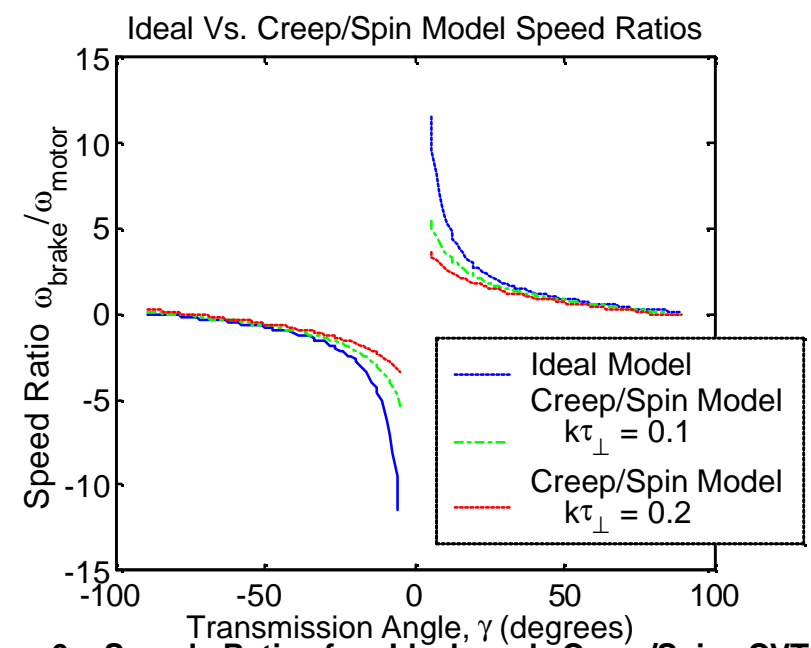

Figure 6. Speed Ratio for Ideal and Creep/Spin CVT models

Applying (16) to (14) and (15), Gillespie also determines the sideslip angle $\alpha$ for the spherical CVT.

$$
\tan (\alpha)=\frac{k \tau_{\perp}\left(\sin ^{3} \gamma+\cos ^{3} \gamma\right)}{1+k \tau_{\perp}\left(\cos ^{2} \gamma \sin \gamma-\sin ^{2} \gamma \cos \gamma\right)}
$$

for $0<\gamma<\pi / 2$ and

$$
\tan (\alpha)=\frac{k \tau_{\perp}\left(\sin ^{3} \gamma-\cos ^{3} \gamma\right)}{1+k \tau_{\perp}\left(\cos ^{2} \gamma \sin \gamma+\sin ^{2} \gamma \cos \gamma\right)}
$$

for $\pi / 2<\gamma<\pi$. Figure 7 shows how the sideslip angle varies with the transmission angle at different fixed lateral loads. The model predicts some $\alpha$ variation with $\gamma$ whose magnitude is largest at multiples of $90^{\circ}$ and whose magnitude is smallest at odd multiples of $45^{\circ}$.

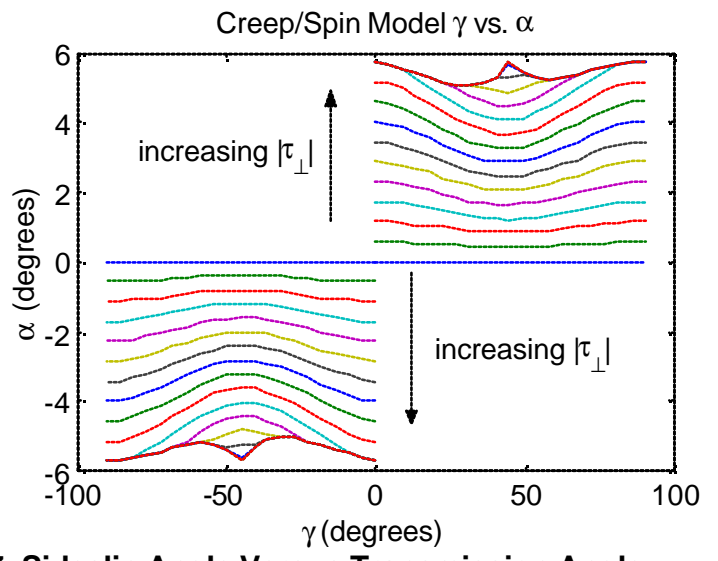

Figure 7. Sideslip Angle Versus Transmission Angle

The theoretical creep/spin model predicted sideslip angle $\alpha$ plotted against $\gamma$ for larger lateral loading as we move toward the top and bottom of the figure, where gross slip would occur.

Figure 8 shows the variation of $\alpha$ with the lateral torque at the transmission angles corresponding to largest and smallest magnitudes of $\alpha$ in Figure 7. Sideslip angle appears to be very linear for small values of $k \tau_{\perp}$ (though the relationship isn't actually linear) and it does go to zero when the lateral torque is zero.

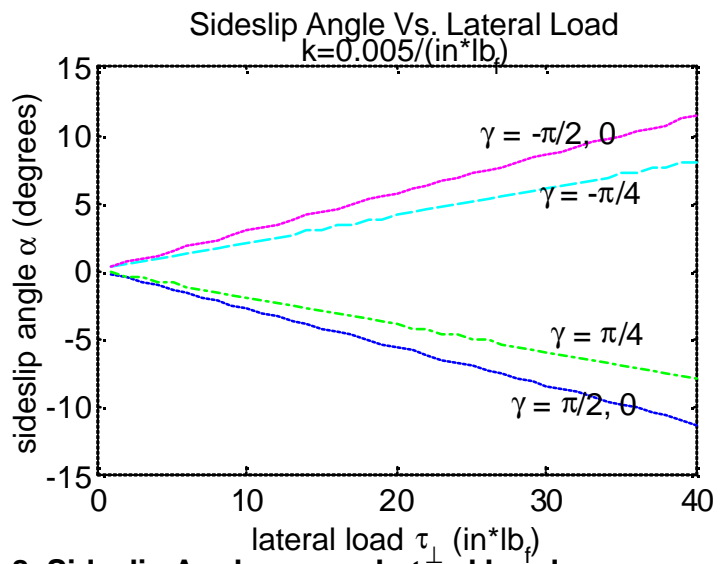

Figure 8. Sideslip Angle versus Lateral Load

It is these creep/spin predictions for speed ratio and sideslip angle that we compare to data collected on an experimental CVT.

\section{EXPERIMENTS AND EXPERIMENTAL MODEL}

To test the validity of the creep/spin predictions for speed ratio and sideslip angle, we applied a constant speed on the motor side of the transmission and a constant torque load on the brake side over a range of transmission angles. As shown in Figure 9, we added several pieces of hardware to a spherical CVT to actuate it and collect the data that would allow us to evaluate the creep/spin model.

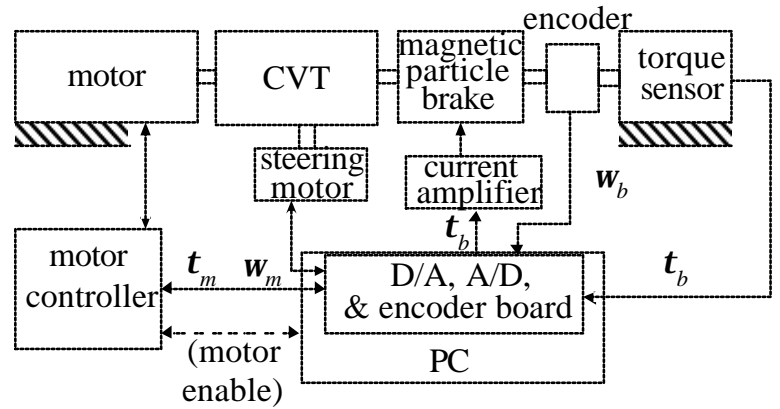

Figure 9. CVT Hardware Diagram

\subsubsection{Bond Graph}

The bond graph shown in Figure 10 models the CVT. The parameters shown in the bond graph are

$R=$ radius of sphere

$r=$ radius of drive rollers

$r_{s}=$ radius of steering rollers

$\mu=$ contact patch Coulomb friction coefficient

$a=$ contact patch half width

And we can define the model parameter $k$ mentioned in section 2.2.2 such that

$$
k=(a / R) /(r \mu N)
$$

$k$ will have units of $1 /$ torque. We choose $k$ this way to collapse all of the parameters affecting the speed ratio and sideslip angle into one constant. (Note that not all of the parameters affecting $k$ are easily measured. E.g., Coulomb 


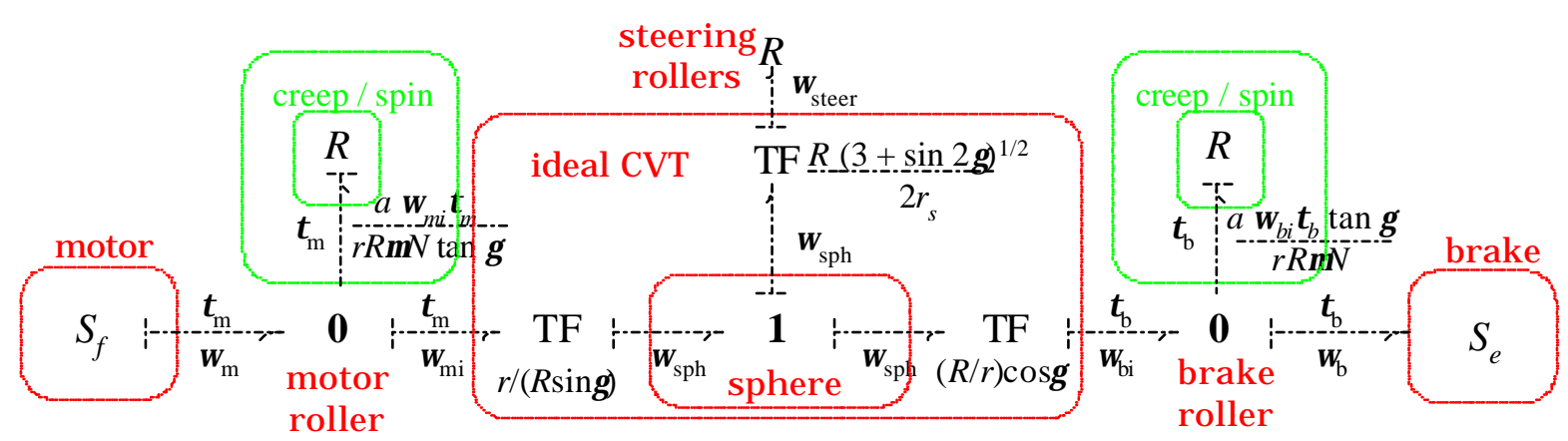

Figure 10. Bond Graph of CVT Experimental Model

friction between bowling ball spheres and roller blade wheel drive rollers varies considerably with testing conditions.) For the system tested, our measured parameter yield

$$
\begin{aligned}
k & =(0.275 / 2) / 4.25) /((3.150 / 2) * 0.7 * 53.2) \\
& =0.000552 /(\mathrm{in} \cdot \mathrm{lbf})
\end{aligned}
$$

The bond graph representation of the experimental CVT allows us to discuss the creep/spin losses in the system as flow losses at the 0 -junctions. These allow us to model the slip that occurs at the drive rollers so that we have the actual velocity of the motor shaft and the velocity lost due to creep- and spinrelated slip in the drive roller contact patch. The difference between the real motor velocity and this slip loss gives us the "ideal" velocities $\omega_{m i}$ and $\omega_{b i}$ going into the "ideal CVT" shown in the bond graph.

The torque and velocity leaving the 0 -junctions enter this "ideal CVT" - a 1-junction connected to three transformers. Conceptually, the 1-junction is the sphere and the transformers connect it to 1) the motor roller, 2) the steering rollers (losses not examined here), and 3) the brake roller. The moduli of these transformers vary only with the transmission angle, $\gamma$.

The brake and motor sides of the bond graph mirror one another. We model motor (run in velocity mode) as a flow source and the magnetic particle brake as an effort source.

\section{DATA PLOTS AND DISCUSSION}

\subsection{Data Range}

At each nominal transmission angle $\gamma$, data was taken by running through a set of brake torque settings designed to give a fairly even range of $\tau_{\perp}$. This was done at constant motor speeds of 4 rotations/sec and -4 rotations/sec (about \pm 25 radians/sec). The range of $\gamma$ was $[-90,-10]$ n $[10,90]$ a $5^{\circ}$ increments and $\tau_{\perp}$ on $\pm[0,40]$ in $\cdot \mathrm{lb}_{\mathrm{f}}$. It turns out that getting data anywhere near $\gamma=0^{\circ}$ (where the transmission axis passes through the motor roller and where, in theory, $\omega_{b} / \omega_{m}= \pm \infty$ ) quickly caused gross slip at the motor roller. Also, due to friction, it was not generally possible to achieve $\tau_{\perp}$ near 0 in $\cdot \mathrm{lb}_{\mathrm{f}}$. Figure 11 shows the positive and negative data regions

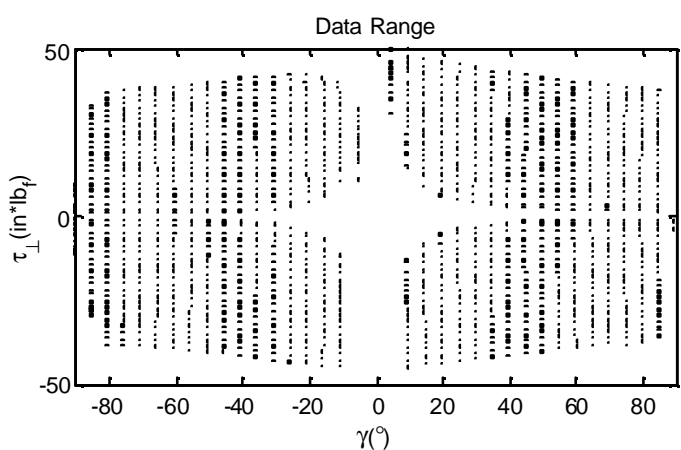

Figure 11. Data Range for $\omega_{m}= \pm 4$ rotations $/ \mathrm{sec}$

\subsection{Sideslip Angle Plots}

The measure of spherical CVT slip that is most easily understood in terms of the translational CVT analogy is the sideslip angle $\alpha$ (see section 2.2).

\subsubsection{Sideslip Angle versus Lateral Load}

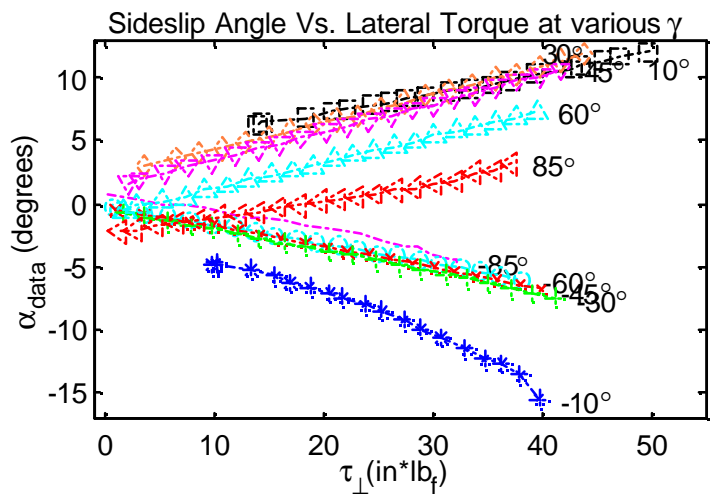

Figure 12. Sideslip vs. Lateral Load, selected $\gamma$

The sideslip angle increases linearly fashion as we increase the lateral load.

Figure 12 shows that the relationship between the sideslip angle and the applied lateral load is fairly linear, just as the creep/spin model predicts (Figure 8).

\subsubsection{Sideslip Angle versus Transmission Angle}

The characteristic shown in Figure 13 does not show the same curvy nature as the one predicted by the creep/spin model (Figure 7). Instead, in the experimental tests, we see 
more slip in the region near where the motor is forced to drive the brake at higher speeds but not where the brake velocity is much lower than that of the motor. On way to interpret this is that we see more slip when the motor roller's contact patch is dominated by spin (rotation in the contact patch) and less when it is dominated by creep (slight longitudinal slip in the contact patch). That is, when the motor is applying power through a contact patch which is swirling underneath it, more slip occurs.

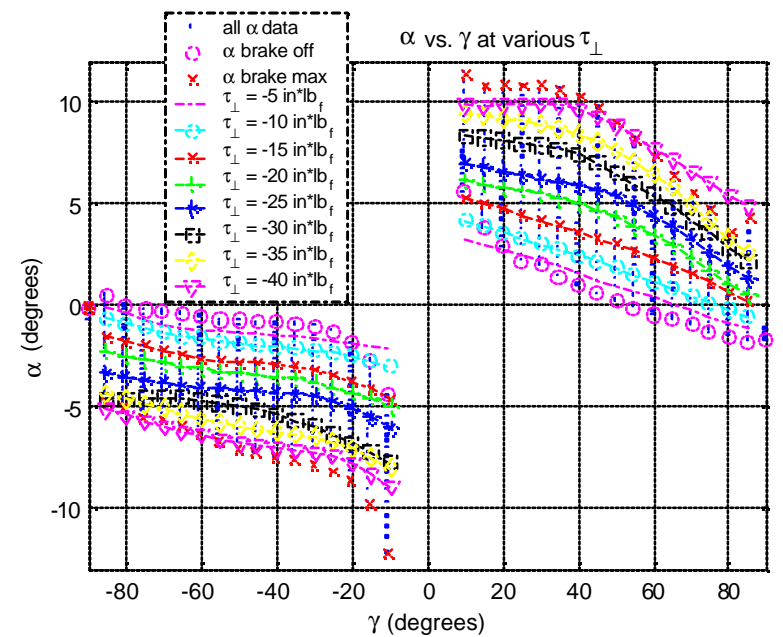

Figure 13. Experimental Sideslip Angle versus Transmission Angle, $\omega_{m}<0$

Dependence on $\gamma$ does not display the slight added curvy characteristic the model does. This is a minor point since that characteristic is small in the previous plot. More interesting is that the experiments showed increased slip as we move from $\gamma= \pm 90^{\circ}$ toward $\gamma=0^{\circ}$, which the model does not.

An oddity we note in this figure is that the sideslip angle $\alpha$ dips past $0^{\circ}$ (changes sign) at low values of $\tau_{\perp}$ near $\gamma=$ $\pm 90^{\circ}$. Strictly speaking, this implies that the brake roller is rotating faster than we would expect in the ideal case, which is really not very likely. What is more likely here is that this is a result of the transmission angle not being exactly what it should be according to the steering roller position. In effect, if $\gamma$ were actually larger than it should ideally be, then we would observe larger brake velocities than at the nominal $\gamma$ and this would result in the behavior we see in $\alpha$ above.

\subsection{Speed Ratio Plot}

In terms of typical transmission applications coupling the one rotating shaft to another, the most obvious measure of a CVT is its speed ratio. In this section we compare an actual speed ratio $T_{b / m}=\omega_{b} / \omega_{m}$ to the "ideal" speed ratio given by (7) and to the predictions of the creep/spin model as given by (14) and (15).

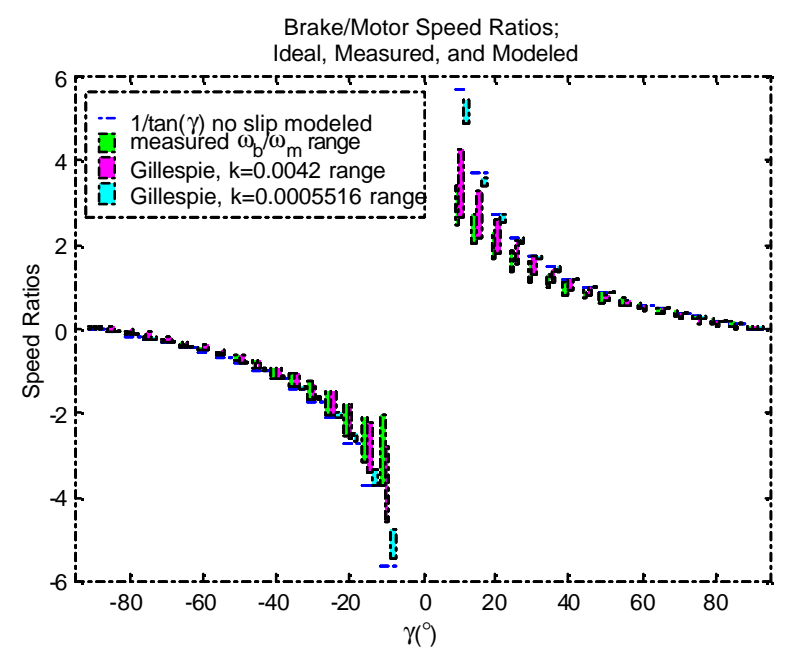

Figure 14. Various Speed Ratios

Here we have speed ratios shown for several different cases. Horizontal lines are ideal (no slip) speed ratios. Also shown are bars showing the speed ratio range (over the applied lateral torques) for three cases: 1) as obtained in the experiments; 2) as predicted by the creep/spin model using a "best fit" value for the model parameter $k ; 3$ ) as predicted using the $k$ derived from measuring the quantities in the expression for nominal $k$ (as given in (19)).

Figure 14 shows the various speed ratios of the spherical CVT. It emphasizes how closely the range of creep/spin predicted speed ratios overlay the measured speed ratios for our applied lateral loads, when the appropriate model parameter $k$ is chosen. First, the horizontal lines mark the ideal speed ratio predicted by the model accounting for no slip. The leftmost bar at each transmission angle shows the actual range of speed ratios observed when we applied our range of lateral loads in the experiments. Then, the corresponding predicted ranges of speed ratio for two $k \mathrm{~s}$ are shown. The middle bar at each $\gamma$ shows the speed ratio range for $k=0.00421 /\left(\mathrm{in} \cdot \mathrm{lb}_{\mathrm{f}}\right)$ as determined by a least squares fit of the data to the model in (14), minimizing the squared difference between predicted and measured sideslip angles. This is quite a bit higher than the second value for $k$ described in (19) and computed by (20), 0.000552/(in.lbf), based purely on measured physical parameters. The rightmost bar at each $\gamma$ shows the predicted speed ratio range for the creep/spin model with that value of $k$. We can see that it tends much closer to the ideal prediction.

But the overall shape of the model predictions is sufficiently similar to the actual CVT behavior that it is still useful, as we will see in the resteering experiments in section 5. We can see that, except for quite near $\gamma=0^{\circ}$, the creep/spin model covers very much the same range as the actual speed ratios, though it does tend to predict a higher speed ratio.

\subsection{Power Use in the Spherical CVT}

\subsubsection{Theoretical Efficiency}

The model shown in Figure 10 depicting losses only due to creep and spin allows us to determine an expression for CVT efficiency as a function of the sideslip angle. Defining 
efficiency $\eta$ in terms of motor power and creep/spin power losses,

$$
\eta=\frac{P_{m}-P_{\text {loss }}}{P_{m}}
$$

we find

$$
\eta=\frac{\tan (\gamma)}{\tan (\gamma+\alpha)}
$$

Which describes CVT efficiency accounting for power losses only due to creep and spin at the drive rollers.

\subsubsection{Measured Efficiency}

We can directly measure the power losses in the experimental CVT from experimental torque and speed data to compute an efficiency including other losses, such as rolling losses at the drive and steering rollers.

Figure 15 displays the efficiency predicted by the creep/spin model (circles) (at the "best fit" $k$ ) for measured lateral loads and transmission angles and the actual experimental efficiency of the CVT (dots). We show this for an entire set of experimental data. While larger lateral torques result in lower CVT efficiencies, the model consistently predicts a higher efficiency than was actually realized. We expect this as the creep/spin model accounts only for slip losses in the drive roller contact patches. The actual CVT looses power overcoming rolling resistance in the drive and steering rollers, lateral slip in the steering rollers, et cetera.

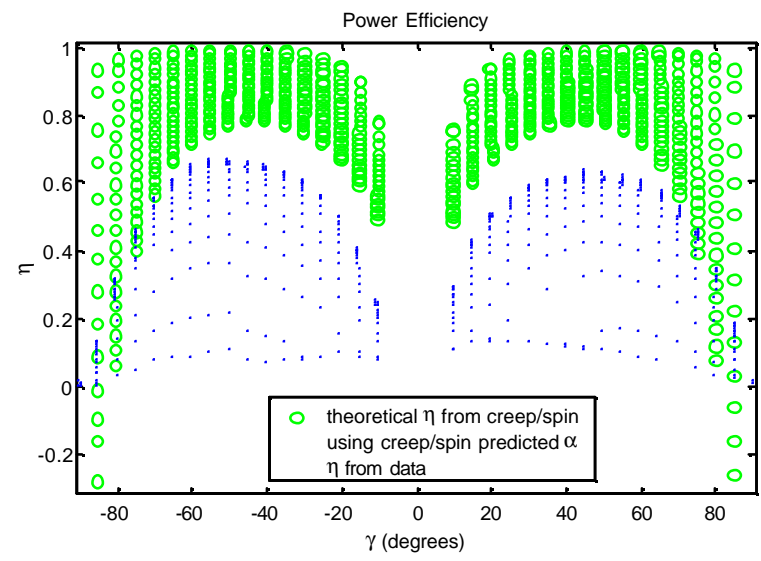

Figure 15. Measured Eficiency from Data and Creep/Spin Predicted Efficiency

We show the efficiency measured from the data (circles) and the efficiency predicted by the creep/spin model applied to the sideslip angles predicted by the creep/spin model for the same data points.

\section{RESTEERING CVTS}

Our primary use for CVTs in cobots uses a particular CVT speed ratio to constrain some cobot endpoint motion to a desired trajectory. In view of this end and in light of the fact the we know CVTs exhibit slip which alters their nominal speed ratio when loaded, we would like to be able to resteer a CVT exposed to some lateral load so that it still provides the desired transmission ratio and, thereby, ensures that its cobot endpoint follows the desired trajectory.

\subsection{Resteering}

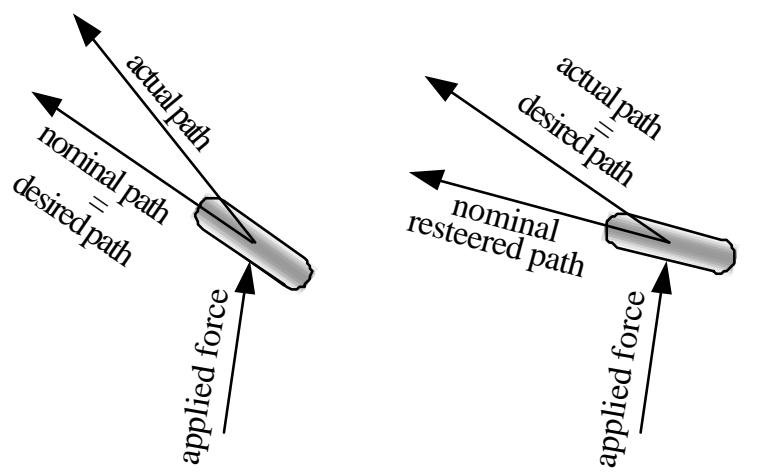

Figure 16. A resteered CVT (right) follows a desired actual path despite lateral forces

The idea of resteering is straightforward. If we have a CVT which is leaving its nominal path, as the CVT of Figure 4 above is, then we wish to change its axis so that, even with the applied lateral forces, it remains on its nominal path. Figure 16 shows a disk CVT before (left) and after (right) resteering it so that its new actual path is the same as the previous nominal (desired) path.

We can determine an appropriate angle to which a resteered CVT must be sent to maintain its desired trajectory using either Gillespie's kinematic creep and slip model [9] or by doing inverted lookup using the experimental CVT data taken. By using a model or lookup data to predict the amount of slip in an open-loop CVT, we can determine the amount of feed-forward steering needed to "pre-correct" for the slip and keep the CVT on its nominal path. In addition, the application of resteering provides us with a good test of the effectiveness of the model predictions in accurately controlling a CVT.

\subsection{Experimental Resteering Data}

We tested our CVT's ability to produce desired transmission ratios under varying lateral loads. The test set loads tested were $\tau_{\perp}=0,10,20$, and 30 in $\mathrm{lb}_{\mathrm{f}}$, nominally, from which we ended up with loads $2-3$ in $\mathrm{lb}_{\mathrm{f}}$ higher from frictions in the system. As we would expect, all of the models worked well at low lateral torques, so we show only on the highest lateral load data. At each loading, we asked for speed ratios $\omega_{\mathrm{m}} / \omega_{\mathrm{b}}=\{0.4,0.5,0.8,1.0,1.25,2.0,2.5\}$, (geometrically symmetric about 1.0).

For each test set, we tested five cases.

- The "ideal" case of $\omega_{\mathrm{m}} / \omega_{\mathrm{b}}=\tan \gamma$.

- The Gillespie model where

$$
\omega_{\mathrm{m}} / \omega_{\mathrm{b}}=\frac{\sin \gamma-k \tau_{\perp} \cos ^{2} \gamma}{\cos \gamma+k \tau_{\perp} \sin ^{2} \gamma}
$$

for our testing range. We test this with $k=0.00056 /$ (in $\mathrm{lb}_{\mathrm{f}}$ ), which is its nominal value based on CVT geometry.

- The Gillespie model with $\mathrm{k}=0.00223$, the value based on creating a least squares fit of sideslip angles to the experimental CVT data. 
- The Gillespie model with $\mathrm{k}=0.00505$, the value based on creating a least squares fit of speed ratios to the experimental CVT data.

- Inverted table lookup of speed ratios from the experimental data. This test was to give a baseline for good response and provide something of a measure of the noise in our data.

\subsubsection{Resteering Results}

The following plot is the comparison between models at the highest lateral torque setting. The plot shows the performance of all five models over the range of requested speed ratios. The vertical axis is the relative error in percentage

$$
\% \Delta \text { error }=\frac{\left(\omega_{\mathrm{m}} / \omega_{\mathrm{b}}\right)_{\text {requested }}-\left(\omega_{\mathrm{m}} / \omega_{\mathrm{b}}\right)_{\text {achieved }}}{\left(\omega_{\mathrm{m}} / \omega_{\mathrm{b}}\right)_{\text {requested }}}
$$

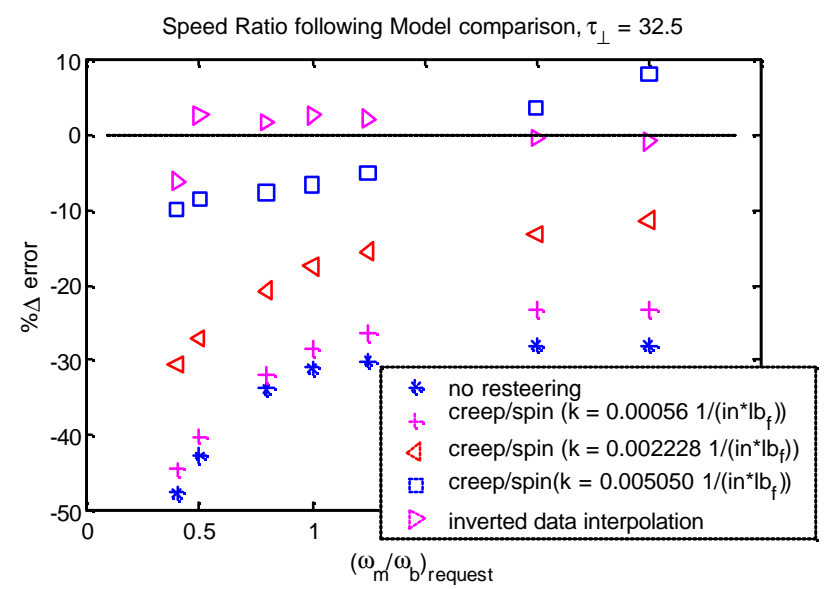

Figure 17. Speed Ratio Following Model Comparison, High Lateral Torque

We show all of the tested CVT models trying to achieve the requested $\omega_{b} / \omega_{m}$ speed ratios $(0.4,0.5,0.8,1,1.25,2$, and 2.5$)$ at a high applied lateral torque. Only inverted lookup and Gillespie model $\left(k=0.00505 /\left(\mathrm{in}_{\mathrm{l}} \mathrm{b}_{\mathrm{f}}\right)\right)$ resteering have average errors under $10 \%$.

As Figure 17 shows, a the highest torque tested for resteering, deviation from the requested speed ratio is getting substantial except for the high $k\left(0.00505 /\left(\mathrm{in}_{\mathrm{l}} \mathrm{f}\right)\right)$ Gillespie model and the inverted data lookup, whose errors average $7.1 \%$ and $2.4 \%$, respectively.

We would further expect from Figure 13 and Figure 14 that the tendency of the CVT to deviate from its ideal behavior will increase as we try to attain speed ratios closer to $T_{b / m}=\infty$. Unfortunately, those plots also indicate that to be the region where the creep/slip model is poorest at predicting CVT behavior and is therefore not likely to be as beneficial in resteering CVTs.

\section{OUTSTANDING ISSUES}

\subsection{Sources of Error in the Creep/Spin Model}

As shown in section 5, the creep/spin model allows much better control of the CVT than does the ideal model, but it is still not perfect, as highlighted, for instance, in Figure 14. Possible sources of error in the model include:

\subsubsection{Assumption of Ideal Behavior at Steering Rollers}

It is true that there is good reason to believe there is no significant longitudinal creep in the steering rollers, as they do not support a longitudinal traction, as do the drive rollers. However, the steering rollers definitely support lateral loads. This load will result in lateral creep and this lateral creep means that the transmission angle $\gamma$ will not be exactly what it should. This effect is qualitatively visible during testing, particularly at values of $\gamma$ near $n \pi(n=0,1,2, \ldots)$ where we desire high values of $\omega_{b} / \omega_{m}$, and it must cause deviation from the model's predicted speed ratios. More exploration of transmission angles actually achieved for given steering angles and under loading will be a significant step in properly predicting CVT behavior and explaining the slight oddities in the data such as those seen in Figure 13.

\subsubsection{Drive Roller Contact Patch Modeled as Line}

The actual shape of the contact patch between drive roller and the sphere looks something like an ellipse with its major axis lying longitudinally (parallel to the tractive forces) as shown in Figure 18A. The real contact patch supports the normal force $N$ of the roller blade wheel into the sphere with a pressure distribution varying from some likely maximum near the center of the ellipse to zero at the ellipse's perimeter. The model in [9] assumes a line contact as shown in Figure 18B with constant pressure (force/length) along the line.

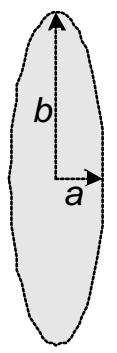

A

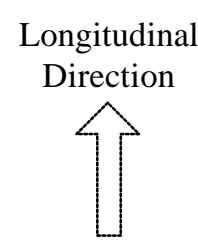

Figure 18. Real vs. Creep/Spin Model Contact Patches The actual contact patch $(A)$ is more ellipsoidal and supports a more complex pressure distribution than the line contact $(B)$ that is modeled in the creep/spin model.

Clearly, this may be a fairly significant simplification. The line model does capture some longitudinal creep and some spin, but it is very likely that the creep and spin in the actual contact patch is different. Particularly, spin in the actual contact patch is probably more dependent on the length of the major axis of the contact patch, whereas the line model is characterized in terms of the minor axis length only.

\subsubsection{Normal Force Variation}

The creep/spin model assumes that the normal force holding the drive rollers against the sphere is constant. 
However, direct observation reveals that the normal force varies both with the applied lateral torque and with the transmission angle $\gamma$.

Analytical results by Songho Kim shows that variation in normal forces results due to CVT geometry. A pair of lateral torques by the two drive rollers generates some combined lateral force and normal force differential in the steering rollers. Assuming there exists a relationship in the distribution of lateral force as function of normal forces on the steering rollers (size of contact patch), variations in normal forces result in the drive rollers. There exist lateral forces on the drive rollers as well, but they are significantly smaller than the drive roller tractive forces.

The knowledge about the lateral forces in the steering roller contact patches may allow exploration of the contact mechanics at the steering rollers. If we can model the slip at the steering rollers, we will have a more accurate model of the sphere's transmission axis, the primary determinant of nominal CVT behavior, as described in section 6.1.1.

\subsection{Further Work on CVT design}

In this work we have compared actual spherical CVT behavior to model predictions and improved the ability of a CVT to attain a desired speed ratio by resteering it.

Further steps in design and use of CVTs could include:

\subsubsection{Eliminating Sources of Error in Creep/Spin Model}

The sources of potential error discussed in section 6.1 are likely addressable via a more sophisticated contact mechanics and kinematic models.

\subsubsection{Exploring Drive and Steering Roller Materials}

Significant continuous power transmission will require tougher materials than the CVTs constructed with roller blade wheels and bowling balls. Quite simply, both of these components are insufficiently robust against normal abrasion and tend to wear down over time.

It may be fruitful to employ different roller materials at the drive and steering rollers. For instance, the steering rollers and driver rollers are doing different jobs. The optimum materials for each task may not be the same.

Similarly, harder roller material would seem to be a benefit all around as they would reduce the size of the contact patch, decreasing losses due to spin. In addition, smaller contact patches reduce steering effort.

\subsubsection{Drive and Steering Roller Shape}

The current use of roller blade wheels as drive and steering rollers results in a roughly elliptical contact patch with the spherical bowling ball. It may be desirable to change the shape of these rollers in order to optimize the major and minor axis lengths, or even make them equal (resulting in a circular contact patch).

We might also alter roller size by optimizing radii versus various metrics. For instance, for high torque transmission applications, having larger drive rollers reduces the needed tractive force in each contact patch, reducing creep and increasing the torque that can be transmitted before gross slip occurs.

In fact, while designing both drive rollers to have the same radius preserves a symmetry in the CVT, it may be useful to design CVTs with a different radius for the motor roller and brake roller. For an application using a fast, but lowtorque motor and low brake speed requirements, we might use a CVT with a small motor roller and larger brake roller to achieve higher maximum brake torques.

At any rate, exploring these problems by analyzing the contact patch mechanics analytically and numerically and perhaps building a test bed to do low level rolling contact experiments seems the likely route to the next step in advancing spherical CVT design.

\section{CONCLUSIONS}

In this work, we focused on comparing a theoretical model of a spherical continuously variable transmission (CVT) accounting for kinematic creep and spin at the CVT's drive rollers (Gillespie's creep/spin model 9]) to an actual CVT, both to better understand CVT function and to determine the utility of the theoretical model in predicting CVT behavior in applications. We built an experimental hardware and software setup allowing us to subject a physical CVT to different lateral loads at a variety of transmission angles. We collected data from this experimental CVT setup to determine the ability of the creep/spin model to predict the speed ratio and sideslip angle for the transmission.

We introduced a bond graph model of the spherical CVT, accounting for both the kinematic creep and spin losses of Gillespie's model as flow losses and various other frictions in the system such as rolling resistance as effort losses. We believe this representation is useful in examining different components of the CVT and we used it to investigate transmission losses in the spherical CVT due to creep and spin. We derived an expression for the CVT's power transmission efficiency, $\eta$, as a function of the sideslip angle. We found that, while the creep/spin model showed the same basic shape of efficiency as the actual CVT, it the tended to underpredict losses as it did not account for losses due to rolling resistance in the drive and steering rollers.

As a check on the model in a practical application, we tested the utility of the creep/spin model to allow corrective resteering of the CVT transmission angle to ensure that it provided the desired speed ratio under a known lateral load and we compared this to an unresteered CVT and resteering attempts made by interpolating within the collected data. We found that, by fitting a model parameter to observed data, the creep/spin model could be practically used to resteer CVTs.

We concluded with a brief discussion of outstanding issues with the spherical CVT and suggestions for further research. 


\section{REFERENCES}

1. W. Wannasuphoprasit, P. Akella, M. Peshkin, J. E. Colgate. "Cobots: A Novel Material Handling Technology" Proceedings of IMECE. 1998

2. R. Brent Gillespie, J. Edward Colgate, Michael Peshkin. "A General Framework for Cobot Control" 1999 International Conference on Robotics and Automation. Detroit, MI.

3. Tatsuhiko Goi, Koji Kawakami, Eiichi Yamakawa, Hirohisa Tanaka. "Variable Rotor Speed Transmission With High Speed Traction Drive" American Helicopter Society 55th Annual Forum, Montreal, Quebec, Canada. May 25-27, 1999.

4. Michael A Kluger and Douglas R. Fussner. "An overview of Current CVT Mechanisms, Forces and Efficiencies" 1997 Transmission and Driveline Systems Symposium, SAE International Congress and Exposition Detroit MI. February 24-27, 1997

5. Tejinder Singh and Satish S. Nair. "A Mathematical Review and Comparison of Continuously Variable Transmissions" SAE Worldwide Passenger Car Conference and Exposition Dearborn, Michigan. September 28 - October 1,1992

6. Johnson, K. L. Contact Mechanics. 1985. Cambridge University Press.

7. Carl A. Moore. Continuously Variable Transmission for Serial Link Cobot Architectures Master's Thesis. Northwestern University. March, 1997.

8. Carl A. Moore, Michael A. Peshkin, J. Edward Colgate. "Design of a 3R Cobot Using Continuously Variable Transmissions" 1999 International Conference on Robotics and Automation. Detroit MI

9. R. Brent Gillespie, J. Edward Colgate, Michael Peshkin, Carl A. Moore. "Kinematic Creep in Continuously Variable Transmissions for Cobots" ASME Journal of Mechanical Design. submitted for final publication November 2001. 